\title{
Dépendance à la trajectoire et création d'une trajectoire dans les controverses sur la sphère bioénergétique au Danemark
}

Path dependency and path creation in the controversies on the Danish bioenergy arena

\section{Bente Hessellund Andersen et Michael Søgaard Jørgensen}

\section{OpenEdition \\ Journals}

Édition électronique

URL : http://journals.openedition.org/economierurale/4741

DOI : $10.4000 /$ economierurale. 4741

ISSN : 2105-2581

\section{Éditeur}

Société Française d'Économie Rurale (SFER)

Édition imprimée

Date de publication : 15 décembre 2015

ISSN : 0013-0559

\section{Référence électronique}

Bente Hessellund Andersen et Michael Søgaard Jørgensen, « Dépendance à la trajectoire et création d'une trajectoire dans les controverses sur la sphère bioénergétique au Danemark », Économie rurale [En ligne], 349-350 | septembre-novembre 2015, mis en ligne le 01 janvier 2017, consulté le 08 mai 2019. URL : http://journals.openedition.org/economierurale/4741 ; DOI : 10.4000/ economierurale. 4741

Ce document a été généré automatiquement le 8 mai 2019.

(c) Tous droits réservés 


\section{Dépendance à la trajectoire et création d'une trajectoire dans les controverses sur la sphère bioénergétique au Danemark}

Path dependency and path creation in the controversies on the Danish bioenergy arena

Bente Hessellund Andersen et Michael Søgaard Jørgensen

La bioénergie suscite des controverses dans le monde entier. Celles-ci ont trait aux aspects sociaux et environnementaux ainsi qu'aux questions méthodologiques. L'un des points le plus débattus concerne la façon dont les interactions entre production de denrées alimentaires et production de bioénergie se répercutent sur la disponibilité de la nourriture à l'échelle locale et mondiale. Ainsi, Kuchler et Linner (2012) décrivent comment des organisations internationales tentent de dresser un tableau plus positif qu'il ne l'est réellement des bienfaits socio-économiques macro qui découlent des échanges internationaux de biomasse, et d'assurer la faisabilité de la production de biocarburants. L'importance d'un environnement naturel en bonne santé et du non-épuisement des sols constitue un autre sujet de préoccupation exploré, entre autres, par Fargione et al. (2010). Ces auteurs ont examiné le changement d'affectation des terres induit par les biocarburants et son impact sur le sol, l'air et l'eau. D'autres controverses proviennent de désaccords sur la méthode permettant d'évaluer si la production et l'utilisation de la biomasse à des fins énergétiques contribuent à la réduction ou au contraire à l'accroissement des émissions de gaz à effet de serre (GES). Des études montrent que les méthodes destinées à estimer l'évolution de ces émissions à la suite d'un projet de bioénergie peuvent fortement influer sur les résultats observés. Galik et Abt (2012), par exemple, examinent une étude de cas consacrée à l'influence de différentes échelles et métriques sur l'évaluation des émissions nettes de GES qui proviennent de la cocombustion du bois en Virginie (États-Unis). 
2 Au Danemark également, pays bien connu pour son exploitation intensive de la force éolienne, la bioénergie occupe une place essentielle dans les programmes climatiques et énergétiques de transition vers une société non tributaire des sources fossiles. Dans certains programmes, il est proposé que 50-60\% de l'énergie totale viennent de la biomasse à l'horizon 2050 (IDA, 2009). Actuellement, près de $75 \%$ de ce que l'on appelle les énergies renouvelables est constitué par diverses formes de bioénergie. Cependant, ces objectifs donnent lieu à de vives contestations, qui portent sur les types de bioénergie, les sources, la provenance et les quantités qu'il est acceptable de produire et de consommer. Ces controverses indiquent que la bioénergie était initialement considérée comme un substitut aux combustibles fossiles qui ne posait pas vraiment problème, mais qu'elle a suscité des préoccupations grandissantes au cours des quarante dernières années.

3 Les rôles assignés à la bioénergie étant très ouverts au Danemark, en termes de technologies, d'acteurs, d'institutions, etc., nous avons décidé de nous intéresser ici à leur formation sous l'angle de la constitution d'une sphère bioénergétique ("bioenergy arena ») dans la stratégie danoise de lutte contre le changement climatique, sans nous concentrer uniquement sur le développement d'une technologie ou d'un secteur en particulier. De surcroît, étant donné qu'en général, différents acteurs suggèrent telle ou telle technologie bioénergétique, à une certaine échelle, sans tenir compte d'autres usages de la source de biomasse, il est nécessaire d'envisager cette "sphère bioénergétique » de façon holistique. Le présent article analyse tout particulièrement les controverses récentes induites par l'enjeu sociétal que représente une stratégie danoise pour le climat, et s'efforce de comprendre comment cette stratégie produit des acteurs et des constellations d'acteurs qui cherchent à introduire la bioénergie comme une solution à l'abandon progressif des énergies fossiles.

4 Partant de l'approche de la sphère du développement (Arena of Developpement, AOD), du mécanisme de dépendance à la trajectoire et de création d'une trajectoire pour les évolutions technologiques sur lequel cette approche se fonde, de la théorie de l'acteurréseau et de la théorie de la gouvernance (Jørgensen, 2012), cet article analyse la formation des récentes controverses, au Danemark, sur les rôles de la bioénergie dans le cadre de la stratégie du pays pour le climat. En repérant des conceptions communes et divergentes entre acteurs, et en analysant les mécanismes de dépendance par rapport à la trajectoire et de création d'une trajectoire via la "navigation» et les tentatives des différents acteurs d'inclure ou d'exclure la bioénergie comme composante de cette stratégie, le présent article expose une conception de la transition qui s'appuie sur ce que nous appelons la « sphère bioénergétique » danoise. Cet article a un triple objectif :

- contribuer au développement d'une mémoire sur les controverses liées à la formation de la sphère bioénergétique danoise ;

- proposer un avis critique aux parties prenantes qui souhaitent formuler des stratégies axées sur l'énergie durable et qui pourraient considérer le Danemark comme un pionnier dans le domaine des énergies renouvelables ;

- remettre en question l'idée que la bioénergie constitue une source d'énergie renouvelable durable.

Nous avons nous-mêmes pris part aux controverses sur la bioénergie au Danemark via les travaux de recherche et le dialogue s'inscrivant dans le cadre de l'alliance pour la transition vers une société sobre en carbone («Enabling and governing transitions to a low carbon society ", www.sustrans.dk) sur la période 2010-2014, ainsi qu'au sein de deux 
organisations non gouvernementales (ONG) danoises, l'IDA - The Danish Society of Engineers et NOAH - Friends of the Earth Denmark (ci-après NOAH), qui, ces dernières années, ont respectivement établi un plan pour le climat et un plan pour l'énergie (IDA, 2009 ; Melgaard, 2010).

6 Notre article est structuré comme suit: les sections 2 et 3 présentent les approches théorique et méthodologique, la section 4 donne un aperçu général des rôles de la bioénergie au Danemark, et la section 5 décrit la formation de plusieurs controverses relatives à la sphère bioénergétique. Pour finir, la section 6 se penche sur les caractéristiques des controverses et sur les différents processus de gouvernance qui ont contribué à ces controverses. Elle se clôt par une analyse de la nécessité d'élargir le champ de la sphère bioénergétique danoise à toutes les formes de biomasse.

\section{Approche théorique}

$7 \mathrm{Au}$ Danemark, les controverses actuelles sur la bioénergie mettent en évidence des différences à la fois dans l'interprétation de la situation qui est celle d'aujourd'hui et dans les conceptions de la production et de l'utilisation de la bioénergie demain. C'est pourquoi, selon nous, il est pertinent de recourir à l'approche reposant sur la sphère du développement (« Arena of Development », AOD) pour analyser les controverses à propos de la manière dont les rôles de la bioénergie ont pris corps. Cette approche répond à la nécessité d'une théorie des processus de transition qui appréhende la navigation et les performances des acteurs. Elle peut être décrite comme " plane », car elle ne recourt pas à des classifications préalables des institutions et des structures sociales selon des niveaux et des hiérarchies (Jørgensen, 2012). Elle a été proposée à la place de la théorie de la transition telle que la Multi-Level Perspective ou MLP (voir par exemple Geels, 2004). Celle-ci traite des transitions et de l'effet des interactions et des tensions entre, d'un côté, les acteurs du régime en place, qui disposent de l'intégralité des ressources et qui défendent le statu quo en raison de leurs intérêts particuliers dans le système sociotechnique établi et, de l'autre, les nouveaux arrivants (ou outsiders), relativement faibles, qui s'efforcent de promouvoir dans des niches des innovations aptes à transformer profondément le système. La division des acteurs et des technologies en régimes, macroenvironnements (landscapes) et niches opérée par la MLP limite la sensibilité analytique concernant le rôle des tensions et des conflits à l'intérieur des institutions dominantes, ainsi qu'entre ces institutions, dans leur navigation (Jørgensen, 2012).

8 L'approche AOD privilégie une analyse plus ouverte pour déterminer si, et comment, les préoccupations sociétales se traduisent par des controverses entre acteurs et constellations d'acteurs au sein des régimes, et si, et comment, ces controverses redessinent et transforment les relations établies entre acteurs, institutions, technologies et infrastructures. Cette approche se concentre sur les dynamiques de dépendance par rapport à la trajectoire et de création d'une trajectoire, pour les modes de production et de consommation dominants, et examine sous l'angle des processus la participation des différents acteurs à la transition. La dépendance à la trajectoire naît de la stabilisation des technologies et des institutions dans différents domaines de la production et de la consommation (Hughes, 1987 ; Garud et Karnøe, 2010).

9 L'approche AOD est axée sur les processus dans lesquels les défis sociétaux, en l'occurrence la stratégie danoise pour le climat et l'idéal d'une société sobre en carbone, 
se traduisent par des questions spécifiques au régime. Pour tenter de pérenniser les relations établies entre acteurs, institutions, technologies et infrastructures, les acteurs et les constellations d'acteurs s'emploient à délimiter les frontières du problème et la réponse. En général, cette délimitation a pour finalité de traduire le défi en mesures concordant avec les relations et les intérêts en place. C'est par exemple le cas lorsque des organisations agricoles cherchent à accroître les aides apportées au biogaz issu du lisier en tant que mesure destinée à réduire les émissions de $\mathrm{CO}_{2}$. Néanmoins, ces stratégies de délimitation ne sont pas toujours fructueuses, et elles sont mises en cause par des acteurs faisant partie d'autres régimes ou ne faisant pas partie d'un régime en particulier, tels que les organisations environnementales. Les processus de délimitation, qui visent à faire de défis sociétaux des défis internes à un pays, ou à les mettre de côté, peuvent donc être très précaires et contestés. L'approche $\mathrm{AOD}$ se fonde sur des approches de la théorie de l'acteur-réseau. Une sphère du développement (AOD) englobe non seulement des acteurs humains, mais également un "ensemble hétérogène d'entités, notamment des êtres humains, des technologies, des conceptions et des pratiques provenant, avec leur signification, leur position et leur identité spécifiques, de leur interaction dans des relations au sein de réseaux" (Jørgensen, 2012). Les processus d'alignement et de médiation aboutissent à la création d'acteurs-mondes (actor-worlds) plus ou moins stables. Ces acteurs-mondes sont des réseaux sémiotiques qui produisent en parallèle le champ, les frontières et la dynamique des sphères. La configuration de la sphère, $\mathrm{y}$ compris de ses frontières, est sans cesse façonnée au travers de processus de stabilisation et de restructuration.

10 Les sphères sont restructurées, et leurs frontières peuvent avancer ou reculer en fonction des activités auxquelles participent les acteurs pour tenter de stabiliser, de transformer ou de déstabiliser les acteurs-mondes existants au niveau d'une sphère. Par référence à Latour (1988), Jørgensen (2012) préconise de privilégier les événements réels, tels que la mise sur pied d'une commission nationale, qui pourraient être considérés comme des déclencheurs de transformations. On peut évaluer la dynamique des sphères d'après ces événements, à l'instar des interactions (par exemple dans le débat public), des projets de démonstration et d'autres façons de créer une présence en relation avec d'autres acteurs, allant de conceptions et de mises en récit pour la construction du sens (storytelling) jusqu'à des interventions matérialisées (Jørgensen, 2012). Les acteurs peuvent faire partie de plusieurs sphères en même temps. Les frontières entre et dans les sphères, ainsi qu'entre les acteurs-mondes qui les configurent, peuvent être évaluées à partir de la constitution des frontières via les activités de construction du sens par les acteurs (Jørgensen, 2012).

11 Conjointement avec l'approche AOD, le présent article recourt à d'autres approches théoriques pour l'analyse des aspects des interactions relatifs à la gouvernance à l'intérieur des sphères. Jessop $(2010,2011)$ conclut à l'importance accrue de formes et de niveaux divers de partenariat entre organisations gouvernementales et non gouvernementales dans la gestion des relations économiques et sociales. Il fait référence à différents types de gouvernance: échanges sur le marché, pouvoir étatique hiérarchique, coordination et dialogue reposant sur des réseaux, confiance et solidarité. Le rôle de la gouvernance fondée sur des réseaux est lié à la complexité grandissante des problèmes auxquels les États sont confrontés. Cette complexité impose d'agir dans différents champs sociaux, à différentes échelles et en différents lieux, étant donné que ni la régulation hiérarchique opérée par l'État du sommet vers la base ni le marché en tant 
qu'institution ne peuvent apporter de solutions efficaces. C'est pourquoi, selon Jessop, les gouvernants s'engagent de plus en plus sur la voie de la méta-gouvernance, définie comme délimitant l'auto-organisation des marchés, des partenariats, des réseaux et des régimes de gouvernance (Jessop, 2010, 2011).

\section{Méthodologie}

12 Cet article a pour socle une analyse des récentes controverses suscitées au Danemark par l'utilisation croissante de la bioénergie et les changements socio-matériels dont elle s'accompagne, ainsi que par les plans et visions destinés à favoriser son essor. Nous avons précédemment mis en avant quatre grandes controverses à propos de la formation de la sphère bioénergétique danoise (Jørgensen, Andersen, 2012), sur lesquelles nous nous concentrons également ici :

- conversion à la paille des centrales au charbon ;

- conversion au bois des centrales au charbon ;

- expansion de la production de biogaz issu de l'agriculture conventionnelle et biologique ;

- développement et production de biocarburants destinés aux transports, couplés à la promotion du bioraffinage pour la production intégrée d'énergie, de fourrage, de produits chimiques et de matières.

13 Pour recueillir des données, nous avons recherché dans la littérature les plans d'action publics et privés dans le secteur de l'énergie, les déclarations faites lors de présentations de programmes publics, les examens d'initiatives pour la bioénergie réalisés par d'autres acteurs et les expériences personnelles qui se dégagent des études et des dialogues portant sur les rôles de la bioénergie. Pour chaque controverse, les données sont organisées en une dynamique des actions des différents acteurs et de leurs interactions au cours du temps.

14 S'inspirant de Latour (1992), Jørgensen (2012) souligne l'importance d'intégrer dans l'analyse de la formation d'une sphère les acteurs exclus, ou mal alignés, dont les pratiques sont concurrentes. Nous nous penchons donc non seulement sur les acteurs dominants, tels que les grandes entreprises du secteur de l'énergie, la branche agroalimentaire, les agriculteurs conventionnels et leurs associations professionnelles, etc., mais aussi sur les technologies et les initiatives de gouvernance moins dominantes, ainsi que sur les différents rôles des organisations de la société civile.

\section{Aperçu général des rôles de la bioénergie au Danemark}

Depuis le milieu des années 1970, la bioénergie figure en bonne place dans les plans axés sur les énergies renouvelables au Danemark. De fait, c'est en 1976 que le pays s'est doté de son premier plan alternatif pour l'énergie, en réaction au premier plan énergétique lancé par le ministère du Commerce au printemps de 1976 (Bülow, 1996). Le plan alternatif dénonçait le plan du gouvernement, qui faisait la part belle au nucléaire et aux installations d'électricité centralisées. Il proposait d'utiliser les énergies renouvelables, dont la bioénergie (Blegaa et al., 1976). Jørgensen et Andersen (2012) décrivent plus en détail l'intégration précoce de la bioénergie dans les plans énergétiques danois des années 1970. 

décentralisée de chaleur et d'électricité, ainsi que pour la production d'électricité à partir de paille et de plaquettes de bois. En 1991, il a instauré une taxe sur les émissions de $\mathrm{CO}_{2}$ imputables aux combustibles fossiles. En 1993, un « plan d'action pour la biomasse » a été voté au Parlement (Danish Energy Agency, 1993), imposant aux installations d'électricité centralisées et décentralisées de mettre en œuvre une certaine quantité de paille et de plaquettes. Selon des arguments avancés en 1990, et encore aujourd'hui, pour justifier l'adoption de mécanismes d'aides au profit de la bioénergie, celle-ci est neutre en $\mathrm{CO}_{2}$, elle utilise des déchets d'origine agricole, forestière, domestique ou industrielle, et, de surcroît, elle contribue à la sécurité énergétique et crée des emplois (Bilgrav-Nielsen, 1996).

Depuis de nombreuses années, on considère généralement la bioénergie comme une source d'énergie renouvelable et respectueuse de l'environnement, malgré des objections. Au début des années 1980, les biocarburants ont suscité un débat au Danemark, certains jugeant immoral d'utiliser des cultures agricoles pour produire du carburant automobile alors même que des millions de personnes souffraient de la faim dans le monde. De même, en 1993, avant l'adoption du plan d'action pour la biomasse, l'association des agriculteurs biologiques a fait entrer dans ce débat les inquiétudes à propos de la «fertilité des sols » et de l'amenuisement de la teneur du sol en matières organiques (Jørgensen, Andersen, 2012).

Depuis le milieu des années 1990, lorsque le Conseil danois de la technologie (Danish Board of Technology) et le gouvernement danois (dans le plan d'action officiel pour l'énergie, intitulé Energi 21) ont, pour la première fois, souligné la nécessité d'une production dédiée de cultures énergétiques pour atteindre les cibles énoncées dans les plans (Nielsen et al., 1994; ministère danois de l'Environnement et de l'Énergie, 1996), l'affectation des terres fait débat: quelle proportion allouer à la culture de denrées alimentaires, à la production d'énergie et à la nature ?

19 Au Danemark, la bioénergie est, dans une large mesure, le plus gros contributeur au développement des énergies renouvelables depuis quelques années. Un examen des chiffres révèle qu'en 2013, sur un total proche de 187 pétajoules (PJ), quelque $40 \mathrm{PJ}$ seulement provenaient de l'éolien, 5 PJ des pompes à chaleur, 3 PJ du solaire et $139 \mathrm{PJ}$ (près de $75 \%$ des énergies renouvelables) des diverses formes de bioénergie, dont environ 47 PJ étaient importés. Depuis 2010, la croissance de la bioénergie importée est plus forte que celle de la production danoise d'énergies renouvelables de tous types (Danish Energy Agency, 2014 b).

Selon le plan national actuel, à l'horizon 2020, les énergies renouvelables devraient entrer pour approximativement $35 \%$ dans la production énergétique totale au Danemark, dont plus de la moitié proviendra de la biomasse. L'utilisation de la bioénergie devrait ainsi augmenter et représenter $166 \mathrm{PJ}$ en 2020, ce qui portera sa consommation par habitant à un niveau nettement supérieur à ce qui est jugé possible à l'échelle mondiale (COWI et Université du Danemark du Sud, 2014). Un accord de mars 2012, qui a réuni un large éventail de partis sur la politique énergétique danoise pour 2012-2020, dans le cadre de "la transition vers un Danemark où les énergies renouvelables couvriront les besoins ", visait entre autres à accroître les investissements dans les turbines éoliennes et les installations bioénergétiques, ainsi qu'à faire monter en puissance la production et l'utilisation de la bioénergie (Agreement, 2012).

Économie rurale, 349-350 | septembre-novembre 2015 
21 Cet accord prévoyait notamment une « analyse de la biomasse » axée sur l'utilisation durable de la bioénergie, afin d'évaluer «si les conditions cadres appropriées sont en place pour une utilisation efficiente et écologiquement durable des ressources issues de la biomasse dans le système d'offre énergétique danois. L'analyse doit également évaluer le déplacement $\mathrm{du} \mathrm{CO}_{2}$ " (Mandate, 2012). Plusieurs analyses secondaires ont été réalisées, dont les plus importantes ont consisté en a) l'exploration d'un scénario général relatif aux systèmes énergétiques du futur, b) une étude parallèle, avec divers horizons temporels, de l'empreinte carbone associée aux différents scénarios (sur la base d'une évaluation du cycle de vie et incluant des estimations des émissions liées au changement direct et indirect de l'affectation des terres), et c) une étude des combustibles ligneux importés, qui a examiné les sources d'approvisionnement potentielles, en provenance de différentes régions du monde, et les problèmes de durabilité (Danish Energy Agency, 2014a).

\section{Analyse de la dynamique des controverses sur la sphère bioénergétique danoise}

Cette section analyse la formation de la sphère bioénergétique au Danemark en étudiant la manière dont les controverses mentionnées plus haut sur les changements opérés au niveau de la production et de l'utilisation de la bioénergie ont pris corps.

Nous avons articulé notre analyse autour de chacune des controverses et nous présentons les actions et les interactions des différents acteurs au fil du temps, y compris le rôle joué par la réglementation et par les plans d'action des pouvoirs publics, etc.

\section{Passage du charbon à la paille}

Répondant entre autres à une requête des agriculteurs traditionnels, qui souhaitaient pouvoir vendre les résidus (souvent appelés " déchets ») de leurs cultures, l'État danois apporte depuis 1993 son aide financière au passage du charbon à la paille pour la cogénération centralisée et décentralisée de chaleur et d'électricité. En 1993, un plan d'action sur la biomasse a été voté au Parlement (Danish Energy Agency, 1993). Ce plan imposait aux centrales électriques d'utiliser un certain volume de paille et de plaquettes de bois à partir de 2000. Il a également défini un ensemble de règles et de directives à l'intention des petites installations concernant le passage au gaz naturel ou à la biomasse, ainsi que la priorité à donner à la production combinée de chaleur et d'électricité (PCCE) à partir de la biomasse.

Certains chercheurs et organisations ont souligné qu'il importait de préserver la teneur du sol en matières organiques afin d'en protéger la fertilité et de pouvoir l'utiliser comme puits de carbone (absorption et rétention $\mathrm{du} \mathrm{CO}_{2}$ atmosphérique dans le sol). Ce rôle est en effet compromis lorsque l'on retire de grandes quantités de résidus des sols aux fins de la production d'énergie. L'association danoise de l'agriculture biologique intervient en première ligne pour défendre cette cause. Ses arguments sont corroborés par des analyses à long terme de l'évolution de la teneur en carbone des différents types de sols où la paille a été brûlée, ramassée à d'autres fins ou labourée dans le sol (Vinther, 2011). De plus, les organisations de défense des animaux militent pour une utilisation accrue de 
la paille dans les étables, laquelle n'est plus guère utilisée dans la plupart des élevages traditionnels (Jørgensen, Andersen, 2012).

L'utilisation de la paille pour la cogénération de chaleur et d'électricité recule. Selon les entreprises du secteur de l'énergie, la combustion de la paille pose problème au niveau des fours. Les agriculteurs déplorent cette désaffection et souhaiteraient que la demande de paille se stabilise, comme ils l'ont par exemple expliqué dans un article publié en ligne dans The Engineer (2010) intitulé « Denmark is Europe's largest importer of wood pellets the straw rots in the fields" (le Danemark est le plus gros importateur européen de granulés de bois, et la paille pourrit dans les champs). Dans cet article, le président de l'association des fournisseurs danois de paille précise que «2,4 millions de tonnes de paille supplémentaires qui restent actuellement dans les champs pourraient être utilisées pour la production d'énergie au Danemark ».

\section{Passage du charbon au bois}

Pour inciter à passer du charbon à la biomasse, le Danemark a prévu d'exonérer de la taxe sur le $\mathrm{CO}_{2}$ la production d'énergie à partir de biomasse, partant de l'hypothèse que la biomasse est neutre en termes d'émissions de $\mathrm{CO}_{2}$. Outre la paille produite par les agriculteurs danois, les plaquettes de bois d'origine locale occupaient précédemment une place importante dans les systèmes de PCCE (centralisés et décentralisés), tandis que la récente progression du recours aux combustibles ligneux dans les systèmes de PCCE a été couverte par des importations. La décision prise en 1993 de contraindre les grandes installations de PCCE centralisées de recourir à la biomasse a ouvert la voie à ces importations depuis la fin des années 1990 : les installations de PCCE rencontraient des difficultés techniques pour brûler de la paille, et le Danemark ne disposait pas d'assez de bois pour permettre la transition prévue du charbon à la biomasse.

Le passage de la biomasse produite localement à la biomasse importée s'est amorcé sans susciter de débat public. La plupart des organisations des secteurs de l'énergie et de l'environnement, qui sont engagées depuis longtemps en faveur des énergies renouvelables, se sont déclarées favorables à la bioénergie. Certaines ont toutefois émis des critiques lorsqu'elles ont découvert que cette augmentation était couverte par de la biomasse importée. Depuis 2010, diverses ONG danoises critiquent l'utilisation accrue des combustibles ligneux (voir par exemple Zanchi et al., 2010), qui accentue selon elles les émissions de gaz à effet de serre (GES) car elle nécessite d'abattre en un bref laps de temps plusieurs années de croissance forestière. En effet, les émissions de GES produites par la combustion de biomasse ligneuse ne sont pas intégralement recapturées, et certainement pas instantanément, par les jeunes arbres en pleine croissance (NOAH, 2010 ; CONCITO, 2011; NOAH, 2012). Ces organisations affirment que ces émissions ne sont pas forcément prises en compte par ceux qui considèrent que les combustibles ligneux (conformément à la méthodologie de la Convention-cadre des Nations Unies sur les changements climatiques, $\mathrm{CCNUCC}$ ) sont neutres sur le plan du $\mathrm{CO}_{2}$. Elles ajoutent que les émissions de GES combinées à la diminution du stockage du carbone par les forêts entrainée par le retrait et la combustion de la biomasse, peuvent se révéler supérieures aux émissions résultant de la consommation des combustibles fossiles. Selon elles, cette situation risque de se produire car les volumes de combustibles ligneux augmentent à l'échelle planétaire. Prenant le contre-pied de ces arguments, deux ONG environnementales danoises (Greenpeace et WWF) ont conclu une alliance avec la 
fédération des entreprises du secteur énergétique danois (Danish Energy Association) pour militer en faveur de la transition du charbon à la biomasse, en escomptant que l'offre de matière produite selon des méthodes durables soit suffisante et en mesure d'atténuer les effets du changement climatique (Nordbo et al., 2013).

Il semblerait qu'aujourd'hui l'hypothèse selon laquelle un recours accru aux combustibles ligneux pourrait produire un effet indésirable immédiat sur le climat rallie de plus en plus de partisans au Danemark. Cependant, une alliance entre des chercheurs spécialisés dans les domaines des forêts et des acteurs du secteur de l'énergie affirme (voir par exemple AEBIOM, 2013) que, si l'on se place dans une perspective de long terme, cet impact sera lissé. En s'appuyant sur cet argument, les entreprises du secteur de l'énergie continuent de prétendre que la biomasse qu'elles emploient est quasiment neutre du point de vue des émissions de $\mathrm{CO}_{2}$ (Danish Energy Association et Danish District Heating Association, 2014), et devrait par conséquent ouvrir droit à l'exonération de la taxe sur le $\mathrm{CO}_{2}$. Certaines $\mathrm{ONG}$ et certains chercheurs s'opposent à cette idée, en faisant valoir qu'elle implique que la forêt et des prairies naturelles soient transformées en plantations, avec à la clé de graves conséquences pour l'environnement (Andersen, 2013), et qu'elle repose sur des hypothèses concernant le climat qui ne sont pas valides (voir, par exemple, Illum, 2014 ; Wittrup, 2014).

30 La transition en cours du charbon à la biomasse passe par une augmentation des importations, principalement en provenance des pays Baltes et d'Amérique du Nord, mais aussi des pays en développement, c'est-à-dire des plantations d'Afrique (Energy Supply, 2011). Il existe un désaccord entre la majorité des acteurs, d'une part, qui estiment que les critères de durabilité peuvent assurer une production durable, et l'organisation environnementale NOAH - Friends of the Earth Denmark, d'autre part, laquelle rejette l'idée d'une "production selon des méthodes durables " s'agissant de la bioénergie importée, et y voit plutôt une manière de légitimer le pillage des ressources et de la nature d'autres pays (Andersen, Eriksen, 2008 ; Andersen, 2010).

NOAH et certains chercheurs reprochent au Danemark d'avoir choisi pour sa transition du charbon à la biomasse une trajectoire qui ne peut pas être reproduite par tous les autres pays, à l'heure où le Danemark se pose en « exemple vert » sur la scène mondiale. Puisque tous les pays doivent passer à des formes d'énergies renouvelables, le Danemark ne devrait pas revendiquer le droit d'utiliser de la biomasse provenant du monde entier, d'autant que sa propre production de biomasse par habitant est supérieure à la moyenne mondiale (Wenzel, 2013). Dans toute l'Europe, l'utilisation de la bioénergie devrait augmenter pour atteindre la cible de $20 \%$ d'énergie renouvelable à l'horizon 2020, comme le prévoient les plans nationaux pour les énergies renouvelables. Ce constat a conduit l'ONG FERN et un ensemble d'autres ONG à conclure que : « la demande accrue de biomasse forestière à laquelle il faut s'attendre compte tenu des dispositions actuelles de la directive sur les énergies renouvelables (RED) et des plans d'action pour les énergies renouvelables des États membres sera difficile à satisfaire à partir de forêts gérées selon des méthodes durables » (FERN et al., 2011).

Le rapport sur «l'analyse de la biomasse » danoise mentionnée plus haut reconnaît que : « Il existe un risque grandissant d'effets indirects liés à la demande danoise de biomasse lorsque les demandes mondiale et régionale de bois augmentent. Étant donné l'offre limitée et à court terme de bois issu des coupes d'éclaircie, de produits du bois résiduels, etc., les clients peuvent être "poussés" à acheter des produits qui ne résultent pas de méthodes de production durables » (Danish Energy Agency, 2014 a) 


\section{Le rôle du bois issu de la foresterie locale}

33 à partir des résidus de la production de bois d'œuvre, c'est-à-dire à partir de bois provenant du sommet et des branches des arbres. Dans le débat sur les enjeux climatiques et de durabilité d'un approvisionnement en produits issus des forêts danoises, on se demande s'il est possible de changer les pratiques de la foresterie de manière à accélérer la croissance végétale (et donc à augmenter l'absorption de carbone) en plantant des « arbres-abris » à croissance plus rapide dans les nouvelles plantations d'arbres à feuilles caduques, entre les essences dédiées à la production de bois d'œuvre. Le secteur danois de la foresterie est favorable à cette pratique à condition qu'il existe à l'avenir un marché pour ces arbres, mais craint que cette production ne soit évincée par le bois importé (Danish Forest Association, 2011 ; Bretner, 2011).

Accorder davantage de place aux conifères (à croissance rapide) au détriment des arbres à feuilles caduques dans les plantations nouvelles constitue un autre moyen de renforcer la croissance végétale ainsi que l'approvisionnement du secteur énergétique, comme l'ont fait les plantations de saules à croissance rapide pour la production de combustible ligneux sur les terres agricoles. Un argument, également lié au climat, milite en faveur de cette pratique : elle permet de séquestrer davantage de carbone dans le sol si les arbres sont plantés sur d'anciens champs agricoles dans le cadre de la rotation des cultures (Gylling et al., 2012). Les ONG de défense de la nature et de l'environnement entrevoient à la fois des possibilités et des problèmes dans cette "approche de l'efficience». Les plantations de conifères et de saules à croissance rapide, en particulier, sont perçues comme problématiques car elles favorisent l'appauvrissement de la biodiversité, amenuisent la diversité des paysages et limitent l'accès aux forêts. Une ONG, The Outdoor Council, critique vivement cette approche, qu'elle juge dictée par des préoccupations économiques et trop indifférente à la nature et à l'environnement, et notamment à la nécessité d'améliorer l'accès du public aux forêts (Nørgaard, 2012).

\section{Biogaz issu de l'agriculture conventionnelle et biologique}

Dans le cadre de l'accord sur l'énergie signé en mars 2012 (Agreement, 2012), le Danemark a décidé de subventionner une forte augmentation de la production de biogaz destiné à différents usages (chauffage urbain ou combustible pour les véhicules lourds). Cette aide est consentie à la fois pour la construction des installations et pour la production de biogaz.

\section{Le biogaz issu du lisier}

Le Conseil danois de l'agriculture et de l'alimentation, une fédération agricole, encourage la production de biogaz à partir de lisier, à même selon lui d'atténuer l'impact climatique et d'augmenter l'emploi (voir par exemple Danish Agriculture and Food Council, 2012). Le biogaz est censé soit être utilisé dans les installations locales de PCCE soit être amélioré et distribué via le réseau de distribution du gaz naturel existant. Cependant, cette stratégie de fabrication de biogaz à partir du lisier est remise en cause depuis des années par les organisations de défense de la nature et de l'environnement, car elle peut servir de prétexte à une expansion de l'élevage. 
37 En collaboration avec l'organisation argentine Grupo Reflexion Rural, NOAH a reproché au biogaz issu du lisier de soutenir une agriculture accordant une trop large place à la production animale reposant sur du fourrage importé (d'Argentine pour l'essentiel). Ces organisations avancent que l'évaluation environnementale du biogaz devrait inclure les impacts de la production et de l'utilisation du fourrage pour l'élevage et ne pas considérer que le lisier est un déchet "gratuit» issu de l'élevage (Andersen, Semino, 2009). Cette opinion concorde avec celle des ONG qui remettent en question les fondamentaux du système agricole danois et l'importante production animale dans le pays, où l'agriculture occupe $63 \%$ de la superficie de terre, et se consacre principalement à la production de fourrage (Danish Society for Nature Conservation, 2011).

Le biogaz ne fait pas l'unanimité au sein de la société danoise car il revient cher en raison des substantielles subventions décidées dans le cadre de l'accord de 2010 sur l'énergie (Agreement, 2012). De plus, le processus de fermentation n'est économiquement réaliste que lorsque des végétaux supplémentaires (par exemple du maïs) sont ajoutés au lisier dans des proportions assez importantes, ce qui limite significativement le potentiel d'atténuation des effets du changement climatique (Bredsdorff, 2012 ; Chrintz, 2013).

Les controverses liées à l'impact environnemental de l'agriculture et à l'intensification de la concurrence internationale ont conduit à la formation de la commission danoise pour la nature et l'agriculture (Danish Commission on Nature and Agriculture). Cette commission se composait à l'origine d'un petit nombre de personnes issues des milieux scientifiques ou du monde de l'entreprise. Un groupe de référence comptant environ 35 représentants des organisations du secteur, du marché du travail et de défense de la nature et de l'environnement a également été mis en place. Cette commission a reçu pour mission d'étudier comment le Danemark peut offrir à sa population « une alimentation saine, goûteuse et de grande qualité, et de nature variée, diverse et cohésive " et " contribuer à lutter contre le changement climatique ", pendant que l'agriculture " peut préserver sa part significative de l'emploi et des exportations dans le secteur alimentaire » (The Danish Commission on Nature and Agriculture, 2013).

Le rapport final de la commission décrit une conception dans laquelle les étables sont gérées et réglementées comme des installations industrielles, visant en priorité la maitrise des émissions dans l'environnement local et la fermentation du lisier pour produire du biogaz. La commission recommande de laisser davantage d'espace à la nature en abandonnant (ou en réduisant) la production dans certaines zones agricoles, et de compenser cet abandon en intensifiant les activités agricoles dans les zones dites " solides " (The Danish Commission on Nature and Agriculture, 2013). Le Conseil danois de l'agriculture et de l'alimentation salue cette conception de l'agriculture (Pedersen, 2013), laquelle déréglemente le volume maximal de production par exploitation ainsi que le volume de l'activité d'élevage à l'échelle nationale. Si ce projet est adopté, la réglementation à venir ne s'appliquera qu'aux émissions locales par exploitation. Cette approche est bien accueillie par certaines organisations environnementales, car elle s'accompagne du développement d'un réseau d'aires naturelles et d'une intensification des efforts dans l'agriculture biologique (Danish Society for Nature Conservation, 2013). Les négociations fondées sur le rapport de la commission ont été engagées à l'automne 2013. Peu après, un plan de croissance pour l'alimentation (Growth Plan for Food) a été lancé à partir de certaines propositions seulement de la commission, notamment celle d'encourager l'élevage intensif (Danish Government, 2013). 


\section{Le biogaz produit à partir de fumier vert sur les exploitations biologiques} engrais et veulent éviter d'avoir à utiliser le lisier provenant de l'agriculture conventionnelle. L'autosuffisance suppose d'inclure des légumineuses fixatrices d'azote dans leur plan de rotation des cultures. La production de biogaz à partir de trèfle, de luzerne et d'autres légumineuses fixatrices d'azote est envisagée. Les agriculteurs biologiques affirment également que cette pratique réduira les rejets d'azote dans l'environnement car elle permet d'apporter des nutriments aux cultures lorsqu'elles en ont besoin et sous une forme qu'elles peuvent assimiler facilement. L'association danoise des agriculteurs biologiques est favorable à la production de biogaz à partir de ces cultures afin de permettre aux agriculteurs d'accroître leurs revenus et d'atteindre l'autosuffisance énergétique (Tersbøl, 2009). Cette association s'est battue pour que les mécanismes actuels d'aide financière au biogaz soient revus afin d'éviter que seul le biogaz produit à partir du lisier y soit éligible. Leur demande a été en partie entendue (Agreement, 2012).

De son côté, l'association des agriculteurs biodynamiques (voir Schmidt, 2009) doute que ce soit une bonne idée de produire du biogaz à partir de trèfle et d'épandre ultérieurement le trèfle traité par digestion à titre d'engrais. Les agriculteurs biodynamiques ont observé dans la pratique que la qualité du sol, y compris sa teneur en humus, s'améliore lorsque l'on y ajoute du compost (produit à partir de fumier vert et animal), et que les bénéfices tirés de cette amélioration (teneur en carbone accrue, plus grande variété des substances nutritives et biodiversité supérieure du sol) surpassent les bénéfices procurés par l'énergie obtenue par la transformation de ce même matériau via un processus de production de biogaz (Loehr-Petersen, 2010).

\section{Biocarburants pour les transports}

43 Selon la directive de l'UE sur les biocarburants de 2003, le Danemark était tenu d'utiliser $2 \%$ de biocarburant dans les transports en 2005 et 5,75\% en 2010. Toutefois, en 2004, le gouvernement danois a informé la Commission de l'UE que le pays n'avait pas l'intention d'introduire de biocarburants dans les transports, en invoquant son expérience de l'utilisation de la paille, à cette époque-là, pour le chauffage urbain. Cette réticence a suscité des critiques de la part de la Commission de l'UE en 2006 (Politiken, 2006). En revanche, elle a été bien accueillie par au moins une partie des ONG environnementales danoises et c'est probablement elle qui explique que ces ONG aient réagi, mais seulement quelques années plus tard, à la cible fixée par l'UE pour les biocarburants, bien qu'elles fussent critiques vis-à-vis du concept de biocarburants dans son ensemble. Les activités de lobbying menées en 2007-2008 par les entreprises et les agriculteurs expliquent peutêtre le revirement soudain du gouvernement danois, lequel a décidé d'une cible obligatoire de $10 \%$ pour les biocarburants à l'horizon 2025, avant même que l'UE n'adopte sa directive sur les énergies renouvelables (RED).

\section{Les biocarburants issus des cultures alimentaires}

Entre 2007 et 2009, jusqu'à l'adoption par l'UE de la directive RED et de la directive sur la qualité du carburant (FQD), les ONG au sein de l'UE et au Danemark se sont efforcées de 
faire exclure la cible relative aux biocarburants de la directive, et un ensemble d'organisations ont lancé un appel à un moratoire sur les biocarburants (EcoNexus, 2007). Des mouvements sociétaux et les populations concernées des continents censés livrer des cultures servant à fabriquer des biocarburants à utiliser en Europe ont expliqué avoir constaté une grave détérioration de leurs conditions de vie, sans pour autant que les promesses de création d'emplois et de revenus ne se soient concrétisées; voir à ce sujet Biofuels - A Failure for Africa (Africa Biodiversity Network et al., 2010). Pour les organisations danoises telles que Danish Action Aid, Greenpeace Danemark, NOAH et The Ecological Council, la coopération avec les organisations internationales s'est révélée utile à deux égards : elle les a aidées à étayer leur argumentaire contre les biocarburants en portant à la connaissance de tous des arguments scientifiques et sociaux et, dans le cadre de leur coopération avec les organisations basées à Bruxelles, en faisant entrer les processus de décision de l'UE dans le débat national danois.

Les biocarburants de la première génération produits au Danemark sont de l'éthanol et du diesel au colza fabriqués à partir de céréales complètes. Les biocarburants importés sont principalement du bioéthanol produit à partir de la canne à sucre. Depuis les débats des années 2007-2008, un consensus se dégage au Danemark sur le fait que les biocarburants de première génération issus de cultures alimentaires ne répondent pas aux critères de durabilité. Cependant, l'organisation des agriculteurs danois estime que les critiques formulées à l'encontre de ces biocarburants sont simplistes (Ministry of Climate, Housing and Energy, 2014).

Nul ne peut dire précisément si l'approvisionnement en biomasse auprès des pays en développement améliorera, ou au contraire dégradera, les conditions de vie pour les petits exploitants et la population. Cependant, aujourd'hui, la plupart des ONG s'accordent à considérer que les bénéfices sont minimes alors que les effets négatifs sont gigantesques. Toutefois, les ONG danoises ne sont pas d'accord sur l'utilité de la certification comme instrument de maitrise et de régulation des conséquences sociales et environnementales de l'importation de biomasse. Des ONG telles que la branche danoise du World Wide Fund for Nature (WWF) soutiennent les programmes de certification reposant sur ce que l'on appelle les "tables rondes pour la biomasse durable", semblables à la table ronde pour l'huile de palme durable, et des initiatives similaires pour le soja et les biocarburants (WWF, 2011). NOAH, de son côté, réfute que ces dispositifs permettent d'assurer la durabilité sociale et environnementale, affirmant que c'est l'échelle même de la production et de la consommation qui n'est pas tenable et que les mécanismes de certification risquent en réalité de conduire à une augmentation de la production, car ils servent d'«éco-blanchiment» («green-washing») légitimant la consommation (NOAH, 2008).

Plusieurs analyses mettent en évidence de gros problèmes concernant la qualité des dispositifs de certification et des systèmes d'audit dont ils s'accompagnent (Schlegel et al., 2011). Certains chercheurs danois s'accordent à penser que ces dispositifs ne sont pas à même de limiter l'expansion des superficies de terre consacrées à la production de biomasse (Hermansen et al., 2013). Le WWF reconnait que les critères pourraient être plus pertinents, mais affirme qu'un surcroit de demande, par exemple, d'huile de palme produite dans des régions ne possédant pas une importante biodiversité, constituerait une amélioration, tout en admettant avec pragmatisme que la demande d'huile de palme va doubler avant 2020 (WWF, 2011). 


\section{Les biocarburants de deuxième génération} génération dans son plan d'action national sur les énergies renouvelables témoigne aussi de l'influence exercée par Novozymes. En effet, dans ce pays, le rapport entre le biodiesel et le bioéthanol penche nettement plus en faveur du bioéthanol que dans les autres pays de l'UE. Novozymes participe très activement au débat et exhorte à un renforcement des cibles contraignantes concernant l'utilisation des biocarburants de deuxième génération (voir, par exemple, EurActiv, 2012). Mais, au Danemark, les acteurs économiques ne sont pas les seuls à appuyer les biocarburants de deuxième génération. Lors du débat sur la directive de l'UE relative aux énergies renouvelables, plusieurs chercheurs danois et DONG Energy ont vanté les avantages des biocarburants de deuxième génération. En outre, en dépit des critiques qu'avait essuyées la première génération de biocarburants, plusieurs ONG environnementales n'ont pas fermé la porte aux biocarburants de deuxième génération, à condition toutefois qu'ils soient produits de manière "durable " (Vilby, 2007 ; Danish 92 Group, 2011; The Danish Ecological Council, 2014). Élaborés à partir de résidus végétaux, les biocarburants de deuxième génération n'induisent aucun changement direct ou indirect au niveau de l'affectation des sols. Par ailleurs, NOAH avance que le désir de mobilité est si grand que les biocarburants de deuxième génération issus de résidus végétaux ne suffiront pas à répondre à la demande. Il faudra, à l'avenir, utiliser des cultures alimentaires et d'autres sources de biomasse, comme les cultures énergétiques, le bois et les plantes herbacées. Les enzymes permettant la décomposition des déchets végétaux ligneux sont, elles, déjà en cours de développement. On pourrait ainsi assister à une expansion des plantations dans les pays en développement et, partant, à de nouveaux cas d'accaparement de terres (Andersen, Eriksen, 2007).

51 En outre, le plus grand syndicat danois est favorable aux investissements dans les usines de production de bioéthanol de deuxième génération. La Fédération unie des travailleurs danois (3F) s'est alliée à l'entreprise Novozymes pour faire pression sur le Parlement afin 
qu'un financement public soit alloué à une installation industrielle de production de bioéthanol de deuxième génération à base de paille. L'argument avancé a trait au potentiel d'exportation de cette technologie «verte » et à la création d'emplois qu'elle pourrait induire dans la construction et l'exploitation des installations, évitant ainsi l'importation de granulés de bois (3F et Novozymes, 2011). Une analyse montre néanmoins que la production et l'utilisation de bioéthanol de deuxième génération entraînent des coûts socio-économiques élevés (EA Energianalyse, 2009).

Malgré les solides alliances qui se sont forgées autour des biocarburants de deuxième génération, les chercheurs qui adoptent une approche holistique des systèmes énergétiques critiquent ce type de combustible. Selon eux, la matière première (comme la paille des céréales) ne devrait pas être considérée comme un résidu "gratuit ", car elle pourrait servir à d'autres usages. La biomasse devrait être considérée comme une ressource limitée, qui doit être utilisée de la façon la plus optimale possible. Ainsi, il ne faudrait y recourir que lorsqu'il n'existe pas d'autre solution. C'est le cas, par exemple, pour la fabrication de produits chimiques et de matériaux, ou encore dans les transports (avions et poids lourds) pour lesquels il ne semble pas exister de solution de substitution au moteur à combustion (Lund, éd., 2011).

Pour leurs partisans, les biocarburants de deuxième génération seront la solution jusque vers 2020-2030, dans l'hypothèse où les automobiles équipées d'un moteur à combustion auront disparu d'ici là (The Danish Board of Technology, 2009). De son côté, CONCITO a avancé, dans un rapport de 2009, que la biomasse « durable » ne suffirait probablement qu'à couvrir les besoins de l'aviation en 2030. Par conséquent, aux yeux de ce think tank environnemental, développer l'utilisation des biocarburants pour les voitures individuelles est « contraire aux objectifs climatiques » (Henningsen et al., 2009).

Les critiques portent également sur l'efficacité énergétique du bioéthanol de deuxième génération, qui serait faible sur l'ensemble du cycle de vie, lequel va de la matière première au moteur. Un groupe de chercheurs propose de recourir au biométhanol, qui provient de l'hydrogénation du biogaz, issu de la gazéification de la biomasse, car il offre un meilleur rendement énergétique (Lund, 2011).

\section{Le concept de bioraffinerie pour une production intégrée d'énergie, de fourrage, de produits chimiques et de matériaux}

Le mouvement en faveur des biocarburants de deuxième génération au Danemark s'intéresse depuis peu au bioraffinage, qui, outre des biocarburants, devrait produire des bioproduits et des biomatériaux, du fourrage pour les animaux et d'autres ressources énergétiques. Créée en 2011, la Biorefining Alliance rassemble une entreprise énergétique, une entreprise chimique et une entreprise biotechnologique, ainsi que le Conseil danois de l'agriculture et de l'alimentation. Son objectif est de «promouvoir la position du Danemark dans la chaîne de valeur qui va de l'utilisation durable de la biomasse aux bioproduits». Sa stratégie a été développée dans le cadre d'un projet de recherche à financement public. Ce projet reposait sur des task forces composées de chercheurs universitaires, d'entreprises et de consultants. Il appelle à une intensification de la recherche et du développement dans la filière du bioraffinage, et notamment à des investissements dans un "Bio Hub", qui "garantirait le chemin le plus court possible entre la recherche et la commercialisation, en passant par la démonstration ». L'alliance recommande en outre de constituer un marché des biocarburants de deuxième 
génération et de réduire les coûts de fabrication du bioéthanol de deuxième génération. Cette stratégie indique que, jusqu'ici, seule la production de bioéthanol de deuxième génération en est au stade permettant la production industrielle (Biorefining Alliance, 2012). Le descriptif de l'un des projets de biocarburants de deuxième génération annonçait (lorsqu'il a reçu un financement d'un fonds danois pour la transition verte) qu'«[...] à long terme [...], il vise à mettre au point [...] du fourrage, des produits biochimiques, des ingrédients alimentaires, etc. à base de sucres et de lignine [c'est nous qui traduisons] (Erhvervsstyrelsen, sans date).

DONG Energy, la grande entreprise énergétique membre de la Biorefining Alliance, appuie l'innovation dans les processus de fabrication de biocarburants de deuxième génération. Elle a investi dans l'une des usines pilotes de production de ces biocarburants (voir, par exemple, DONG Energy, 2015). Elle a également financé un projet de recherche mené par deux universités qui ont étudié les possibilités de multiplier par trois la production danoise de biomasse, afin de l'utiliser pour le bioraffinage. Le rapport « The +10 million tons plan» (Gylling et al., 2012) se penche notamment sur les moyens d'éviter l'épuisement du carbone fixé dans la matière organique des sols (en dessous d'une certaine limite), tout en mettant en œuvre davantage de paille pour la production d'énergie et le bioraffinage. Les auteurs proposent de compenser la perte entrainée par le ramassage de la paille en cultivant soit des céréales secondaires soit des céréales dont la paille est plus longue. Ils suggèrent également d'utiliser davantage les cultures énergétiques pérennes dédiées afin que de la matière organique s'accumule dans le sol et, partant, qu'elle capte le carbone. Le biogaz et le bioéthanol pourraient ainsi remplacer $20 \%$ des besoins actuels en gaz naturel et 30-50 \% de ceux en essence et en gazole (Gylling et al., 2012).

Dans son rapport final, la commission pour la nature et l'agriculture se prononce également en faveur du bioraffinage, préconisant une "utilisation intelligente de la biomasse » et « des efforts offensifs pour parvenir à une biomasse durable ». En outre, la commission recommande de stimuler la demande de biocarburants de deuxième génération, et propose de rendre obligatoires les exigences de l'UE et du Danemark concernant l'ajout de biocarburants de deuxième génération à l'essence (Commission on Nature and Agriculture, 2013).

\section{Discussion et perspectives d'avenir}

\section{Dynamique de la sphère bioénergétique danoise}

L'analyse présentée à la section 5 montre que, ces dernières années, la sphère biotechnologique danoise s'est complexifiée et que les conflits sur les stratégies et les visions pour l'avenir s'y sont multipliés. Hier, au Danemark, la bioénergie était produite et utilisée principalement localement. Elle établissait un lien entre l'agriculture locale, l'industrie agroalimentaire locale et la production et la consommation locales de chaleur et d'électricité. Par exemple, une usine de biogaz ou une installation énergétique locales reçoivent de la biomasse locale et desservent la population locale. La priorité accordée depuis peu à l'atténuation des changements climatiques au niveau international et la volonté du Danemark de s'affranchir des énergies fossiles en 2050 plaident pour un recours accru à la bioénergie dans le but de réduire les émissions de gaz à effet de serre. 
59 Les analyses montrent l'intérêt que présente le plan danois pour la transition vers une société sans énergies fossiles aux yeux des entreprises de plusieurs secteurs, des acteurs gouvernementaux et des organisations de la société civile. Lorsqu'ils définissent des initiatives de lutte contre le changement climatique, les entreprises et les pouvoirs publics suivent parfois des approches qui dépendent de la trajectoire. C'est notamment le cas, dans le domaine de la bioénergie, des initiatives reposant sur les granulés de bois destinés aux grandes centrales électriques, ou sur le lisier issu de l'élevage pour la production de biogaz. D'autres initiatives créent une trajectoire et reposent sur le développement de nouvelles alliances intersectorielles. Ainsi, des alliances se nouent entre l'agriculture, la biotechnologie et le secteur pétrolier pour le développement de biocarburants de deuxième génération. Cependant, certains secteurs restent fortement dépendants de la trajectoire.

60 Lorsque l'on analyse les controverses, on s'aperçoit que de multiples acteurs venant d'horizons divers font partie intégrante de la sphère bioénergétique danoise: des entreprises, des organisations professionnelles, des organismes publics, des responsables politiques, des ONG, des chercheurs et des institutions de recherche. Tout comme les flux de matières, les réseaux de savoir et les structures de gouvernance se sont, dans une certaine mesure, internationalisés. Les chercheurs coopèrent à l'international, l'UE régule le rôle de la bioénergie, les programmes de certification de la bioénergie sont internationaux et les ONG du secteur de l'environnement et du développement coopèrent et partagent leur savoir, elles aussi, à l'international.

61 Sur la base de l'analyse détaillée, présentée à la section 5, des quatre controverses relatives à la production et à l'utilisation de la bioénergie, nous étudions ci-après certains aspects de la dépendance vis-à-vis de la trajectoire et de la création d'une trajectoire dans ces controverses, ainsi que le type de processus de gouvernance qui est à l'œuvre dans la sphère bioénergétique danoise : conversion des centrales à charbon en centrales utilisant différentes formes de bioénergie (paille et bois), rôle du lisier et du biogaz en relation avec l'élevage, priorité au développement de biocarburants de deuxième génération et interaction avec le bioraffinage.

\section{La bioénergie dans les centrales électriques}

Les entreprises énergétiques veulent conserver les centrales de cogénération existantes, car elles représentent, selon elles, un facteur de stabilisation au sein du réseau électrique. Elles préconisent en outre le recours à la bioénergie, étant donné que c'est une source énergétique neutre en $\mathrm{CO}_{2}$. L'État encourage ces entreprises à opter pour la bioénergie en leur permettant de réaliser des économies sur leurs coûts énergétiques, via l'exonération de la taxe sur le $\mathrm{CO}_{2}$. Cette conversion, qui s'appuie sur les centrales existantes, constitue un changement dépendant de la trajectoire dans l'infrastructure énergétique existante. Du point de vue de la gouvernance, elle est rendue possible par la combinaison des échanges sur le marché (achats de biomasse), des décisions imposées par l'état (exonération de la taxe sur le $\mathrm{CO}_{2}$ ) et de la formation de réseaux (alliance avec des chercheurs du secteur forestier). Cependant, les critiques formulées par certaines ONG et certains chercheurs à propos des importations de biomasse ont poussé le secteur de l'énergie à entrer dans un processus de gouvernance reposant sur des réseaux, où les ONG et les chercheurs négocient afin d'élaborer des orientations d'après ce que le secteur de l'énergie et des ONG environnementales estiment être la «bioénergie durable». Les 
controverses à propos des programmes de certification témoignent d'un désaccord quant à savoir s'il faut influer sur le niveau de consommation : ceux qui rejettent les critères de durabilité estiment qu'il est plus important d'agir sur le niveau de consommation que de demander que la consommation des pays industrialisés soit en partie couverte par une « production durable » dans les pays en développement.

\section{Du lisier pour le biogaz}

63 Le secteur agroalimentaire, dont l'élevage forme un pan non négligeable, en particulier dans la filière porcine danoise, est favorable à la production de biogaz à partir de lisier. Cette solution est, à ses yeux, durable et contribue aux efforts d'atténuation des effets du changement climatique. L'objectif est de créer un appui envers une stratégie climatique dépendante de la trajectoire au sein du secteur, reposant sur une expansion de l'élevage. Cette stratégie s'appuie sur une alliance avec divers acteurs de l'infrastructure du gaz naturel et, en ce sens, elle est également dépendante de la trajectoire. Grâce à une gouvernance qui repose sur des réseaux intégrant des acteurs politiques, l'organisation des entreprises agricoles a réussi à obtenir des investissements et des conditions d'exploitation favorables pour les installations au biogaz. La commission pour la nature et l'agriculture est un exemple de sphère intermédiaire pour la gouvernance reposant sur des réseaux à laquelle participent différentes catégories d'acteurs. La commission ne s'intéresse qu'aux effets de l'approvisionnement en fourrage sur l'environnement au Danemark, et non à son impact dans d'autres pays. C'est un exemple de rétrécissement des frontières de la sphère bioénergétique au Danemark. Les raisons qui président à la position de la commission et à l'élaboration rapide du "plan de croissance pour l'alimentation " ( Growth Plan for Food») (The Danish Government, 2013), qui n'a pas fait l'objet d'un débat public, semblent être les emplois et les recettes d'exportation que procurent actuellement les produits à base de viande et l'intensification de la concurrence internationale sur le marché de la viande. L'action de la commission conjuguée au plan élaboré par les pouvoirs publics, combine gouvernance étatique hiérarchique et gouvernance fondée sur des réseaux incluant des acteurs du secteur et des syndicats.

\section{Biocarburants de deuxième génération et bioraffinage}

La mise au point d'enzymes par une entreprise de biotechnologie danoise pour la production de biocarburants de deuxième génération relève à la fois de la dépendance vis-à-vis de la trajectoire et de la création d'une trajectoire. Le secteur danois des biotechnologies et l'industrie pétrolière internationale s'alignent ainsi pour inscrire les biocarburants dans une stratégie climatique dépendante de la trajectoire, qui permettra de continuer à utiliser le moteur à essence et le moteur diesel, ainsi que le réseau existant de stations-service. La mise au point d'enzymes permet la poursuite, dans une optique de dépendance vis-à-vis de la trajectoire, de la stratégie des entreprises de biotechnologie qui consiste à développer des enzymes à des fins environnementales (par exemple lorsqu'elles participent aux campagnes du secteur des détergents en faveur du lavage des vêtements à basse température, ce lavage étant possible grâce aux enzymes pour détergents que produisent ces entreprises), même si c'est dans un autre secteur (Novozymes, 2015). Cette stratégie est dans le même temps créatrice d'une trajectoire, car 
elle s'appuie sur les nouvelles relations commerciales entre le secteur biotechnologique et le secteur pétrolier.

Les processus de gouvernance autour des biocarburants au Danemark revêtent trois formes: les entreprises demandent à l'État et à l'UE de renforcer les critères relatifs à l'ajout de biocarburants de deuxième génération à l'essence, afin de soutenir le développement du marché national et international pour ce type de biocarburant. Mais des processus de gouvernance reposant sur des réseaux sont également à l'œuvre. Le rapport de la commission pour la nature et l'agriculture mentionne les biocarburants de deuxième génération, qui ont aussi les faveurs du syndicat des travailleurs de l'agriculture et de l'industrie. Ce dernier a souligné, avec l'entreprise de biotechnologie, les liens entre les biocarburants, l'économie nationale et la création d'emplois.

L'attention que l'on porte depuis peu au concept de bioraffinerie, dont les biocarburants de deuxième génération ne sont que l'un des produits, peut être envisagée comme une tentative d'élargir l'horizon de la sphère bioénergétique. Celle-ci se concentrerait ainsi non plus uniquement sur les biocarburants, qui sont sujets à controverses, mais aussi sur les biomatériaux et les bioproduits chimiques. On pourrait estimer que le bioraffinage, en tant qu'approche de production, crée une nouvelle trajectoire, par rapport à l'approche actuelle de l'industrie chimique, qui recourt essentiellement au pétrole et au gaz comme matière première pour les synthèses chimiques. Cependant, la production de biomatériaux et de bioproduits chimiques ne constitue toujours pas un pan essentiel de la stratégie danoise du bioraffinage.

On pourrait considérer que la promotion du concept de bioraffinerie conjuguée à la priorité donnée aux ressources de biomasse danoises dans le " +10 million tons plan", financé par une grosse entreprise énergétique publique (Gylling et al., 2012), vise à tenir compte des critiques concernant l'importation de biomasse à des fins énergétiques. Or, ce plan ne reprend nullement cette critique à son compte dans son argumentation.

\section{Perspectives}

Cet article a étudié la manière dont des constellations d'acteurs, à la fois établis et nouveaux, agissent et interagissent dans une "sphère du développement " («Arena of development »- AOD), que nous appelons la «sphère bioénergétique ». En considérant la formation des rôles de la bioénergie comme la formation d'une sphère sur la durée, avec de multiples acteurs et processus de gouvernance, plutôt que le développement d'une technologie, d'une niche ou d'un secteur particuliers, et en incluant les actions d'acteurs non historiques dans les analyses, l'approche AOD et la priorité qu'elle accorde à la «navigation » et à la gouvernance peuvent constituer un outil d'analyse pour les acteurs qui réfléchissent aux possibilités et aux obstacles que présente l'inclusion d'autres acteurs dans leur stratégie.

69 L'analyse des quatre controverses relatives à la sphère biotechnologique danoise montre que les thématiques importantes pour la définition de la production et de l'utilisation de la bioénergie ont trait aux questions suivantes :

1. Quelles ressources de biomasse danoises devraient être utilisées pour quels objectifs?

2. Comment évaluer les conséquences climatiques et environnementales du passage de l'énergie fossile à la bioénergie? 
3. Est-il nécessaire de combiner les évaluations des conséquences climatiques et les autres conséquences environnementales du passage de l'énergie fossile à la bioénergie ?

4. Comment conjuguer les aspects environnementaux, sociaux et économiques d'un abandon des énergies fossiles?

5. Dans quelle mesure les projets de bioénergie réduisent-ils les émissions de gaz à effet de serre?

6. Faut-il, et de quelle manière, combiner la transition de la production énergétique à une réduction de la consommation énergétique?

7. Peut-on utiliser de la biomasse importée dans le système énergétique danois en vue d'abandonner les énergies fossiles, et si oui comment?

8. Peut-on parvenir à une utilisation durable de la bioénergie grâce à un approvisionnement en biomasse certifiée?

70 Ces questions sont fortement liées aux désaccords portant sur les stratégies tant dépendantes de la trajectoire que créatrices d'une trajectoire au sein des secteurs de l'énergie, de l'agroalimentaire, de la biotechnologique et des transports. Les organisations et les groupes de recherche dans le domaine de l'environnement ont réussi à introduire une perspective plus critique sur les biocarburants de première génération et sur les importations de biomasse au Danemark. Les organisations environnementales ne sont toutefois pas d'accord entre elles quant au rôle des biocarburants de deuxième génération et quant à savoir si les programmes de certification sont à même de garantir des importations de biomasse durable. Certains acteurs critiquent également l'emploi du biogaz dans le cadre d'une stratégie d'intensification de l'agriculture. Or les emplois et les revenus que procurent actuellement la transformation et l'exportation de viande et de produits laitiers empêchent les entreprises de changer de stratégie et l'État de passer à une réglementation axée sur une agriculture moins intensive.

71 Sur la base d'une modélisation des changements possibles dans la production et la consommation de biomasse en Allemagne, Bringezu et al. (2009) ont montré qu'il est possible de réduire les besoins nationaux en biomasse. La solution passe essentiellement par l'introduction d'une régulation de la demande (amélioration du rendement des carburants, baisse de la consommation d'aliments d'origine animale et réduction du gaspillage alimentaire). Cette modélisation a révélé que ces initiatives pourraient permettre à l'Allemagne de disposer de suffisamment de terres pour produire une biomasse utilisable à différentes fins.

Pour améliorer son autosuffisance vis-à-vis de la biomasse et réduire les conséquences environnementales tant nationales que mondiales, il faudrait que le Danemark réduise sa demande de biomasse en trouvant une autre manière de satisfaire ses besoins alimentaires et énergétiques actuels. Il pourrait, par exemple, changer les modèles de mobilité et les habitudes alimentaires en vue de faire diminuer la consommation de viande et de produits laitiers. La modification des schémas de consommation forme un volet important des plans climatiques et énergétiques de la Société danoise des ingénieurs (IDA, 2009) et de NOAH (Melgaard, 2010). Dans les années qui viennent, nous verrons s'il est possible d'appuyer ces changements dans le cadre d'une stratégie danoise de la biomasse. 


\section{BIBLIOGRAPHIE}

3F and Novozymes (2011). To the Climate-, Energy- and Building Committee (Danish). Online at: http://www.ft.dk/samling/20111/almdel/keb/bilag/97/1049589.pdf

African Biodiversity Network, Ethiopian Society for Consumer Protection, Gaia Foundation (2010). Biofuels - A Failure for Africa. Online at: http://www.africanbiodiversity.org/sites/default/ files/PDFs/Biofuels\%20-\%20A\%20Failure\%20for\%20Africa\%20(ABN,\%20Dec\%202010).pdf

Agreement between the government (the Social Democrats, the Social Liberals, Socialist People's Party) and Liberals, Danish People's Party, the Red-Green Alliance and the Conservative People's Party about the Danish energy politics 2012-2020 (Danish) (2012). Folketingstidende A, 2011-2012, bilag 2 i L 182 Forslag til lov om ændring af lov om fremme af vedvarende energi, lov om elforsyning, lov om naturgasforsyning og lov om Energinet.dk. Online at: http:// www.folketingstidende.dk/RIpdf/samling/20111/lovforslag/L182/20111_L182_som_fremsat.pdf

AEBIOM (2013). Forest Sustainability and Carbon Balance of EU Importation of North American Forest Biomass for Bioenergy Production, Report Prepared By: AEBIOM European Biomass Association, BC Bioenergy Network, U.S. Industrial Pellet Association and Wood Pellet Association of Canada, with the collaboration of: Drax, GDF SUEZ/Laborelec, Essent NV, E.ON Climate \& Renewables, Danish Energy Association, Vattenfall AB. Online at: http://www.aebiom.org/wp-content/ uploads/2013/09/Final-Carbon-Study-Report-AEBIOM.pdf

Andersen B.H. (2010). Food and fuel for Europe (in Danish). NOAH's Forlag. Online at: http:// noah.dk/wp-content/uploads/2011/11/Mad-og-br\%C3\%A6ndstof-til-Europa.pdf

Andersen B.H. (2013). Sustainability bla bla (newspaper comment in Danish). Arbejderen, November 9. Online at: http://www.arbejderen.dk/blog-indl\%C3\%A6g/bente-hesellund/b\%C3\% A6redygtigheds-bla-bla

Andersen B.H., Eriksen S. (2007). Brave new world of fuels (newspaper comment in Danish), Information, September 27. Online at: http://www.information.dk/146967

Andersen B.H., Eriksen S. (2008). Not one step in the right direction - biofuels and sustainability are incompatible (newspaper comment in Danish). Information, April 4. Online at: http:// www.information.dk/157366

Andersen B.H., Semino S. (2009). Pigs and Soya production: a contribution to the planetary inferno, NOAH and GRR. Online at: http://noah.dk/wp-content/uploads/2009/12/Soya-andPigs_folder.pdf

Andersen U. (2012). Danish production of second generation bioethanol demands more support (Danish). Online at: http://ing.dk/artikel/dansk-produktion-af-andengenerations-bioethanol-kraevermere-stotte-133352

Bentsen N. S., Stupak I. (2012). Imported wood fuels. A regionalised review of potential sourcing and sustainability challenges. Department of Geoscience and Natural Resource Management, Faculty of Science, University of Copenhagen.

Bilgrav-Nielsen J. (1996). The integration of environmental concerns into energy policy (in Danish). In Rehling and Ministry of Environment and Energy 2nd office (ed), Ministry of 
Environment and Energy 1971-1996 - conception, development and outward view, Ministry of Environment and Energy, Copenhagen, pp. 111-118.

Biorefining Alliance (2012). Denmark in a Global Bio-Based Society - Do we want to be customers or producers? Online at: http://www.biorefiningalliance.com/uploads/Strategi_UK_online.pdf

Bleegaa S., Hvelplund F., Jensen J., Josephsen L., Linderoth H., Meyer N.I., Balling N.P., Sørensen B. (1976). Sketch for an alternative energy plan for Denmark (in Danish). OOA, Organisationen til Oplysning om Atomkraft.

Bredsdorff M. (2010). Denmark is Europe's largest importer of wood pellets - the straw rots in the fields (in Danish). Ing.dk 20-01-2010. Online at: http://ing.dk/artikel/105889-danmark-er-europasstoerste-importoer-af-traepiller-halmen-raadner-paa-markerne

Bredsdorff M. (2012). Researchers: A boondoggle history using corn to gas (in Danish). Ing.dk 20-04-2012. Online at: http://ing.dk/artikel/forskere-en-molbohistorie-bruge-majs-tilgas- 128557

Bretner M.L. (2011). Personal communication with Marie-Louise Bretner from The Danish Forest Association.

Bringezu S., Schütz K., Arnold F., Merten S., Kabasci, Borelbach P., Michels C., Reinhardt G.A., Rettenmaier N. (2009). Global implications of biomass and biofuel use in Germany - Recent trends and future scenarios for domestic and foreign agricultural land use and resulting GHG emissions. Journal of Cleaner Production, $\mathrm{n}^{\circ}$ 17, S57-S68.

Bülow H.v. (1996). The first years of the Energy Agency (in Danish). Rehling and Ministry of Environment and Energy 2nd office (eds.), Ministry of Environment and Energy 1971-1996 conception, development and outward view, Ministry of Environment and Energy, Copenhagen 97-104. Online at: http://mim.dk/ministeriet/baggrund-og-historie/jubilaeumsskrift/de-foersteaar/

EcoNexus (2007). Call for an immediate moratorium on EU incentives for agrofuels, EU imports of agrofuels and EU agroenergy monocultures (signed by many organisations). Online at: http:// www.econexus.info/call-immediate-moratorium-eu-incentives-agrofuels-eu-imports-agrofuelsand-eu-agroenergy-monocultur-0

Chrintz T. (2013). The climate impact from biomass and other energy sources (in Danish). CONCITO.

CONCITO (2011). Does the use of biomass reduce atmospheric CO2 content? (in Danish). CONCITO. Online at: http://www.concito.dk/udgivelser/reducerer-brug-biomasse-atmosfaerens-indhold$\operatorname{co} 2$

COWI and University of Southern Denmark (2014). Carbon footprint of bioenergy pathways for the future Danish energy system (in Danish). COWI and University of Southern Denmark for the Danish Energy Agency.

Danish Agriculture \& Food Council (2012). Employment effects of biogas in the energy agreement (in Danish). Online at: http://www.lf.dk/ /media/lf/Tal\%20og\%20analyser/Samfundsokonomiske\% 20analyser/2012-03-23\%20Biogas\%20og\%20energiforlig.ashx

Danish Energy Agency (1993). The Biomass Agreement of June 1993 (in Danish). Online at: http://193.88.185.141/Graphics/Energipolitik/dansk_energipolitik/politiske_aftaler/ biomasseaftale_1993.pdf

Danish Energy Agency (2014 a). Analysis of Bioenergy in Denmark (in Danish). Online at: http:// www.ens.dk/sites/ens.dk/files/undergrund-forsyning/el-naturgas-varmeforsyning/ Energianalyser/nyeste/bioenergi_-_analyse_2014_web.pdf 
Danish Energy Agency (2014 b). Energy Statistics 2010 (in Danish). Online at: http://www.ens.dk/ da-DK/Info/TalogKort/Statistik_og_noegletal/Aarsstatistik/Documents/Energistatistik_2010.pdf Danish Ministry of Climate and Energy (2009). L 181 Act on sustainable biofuels (in Danish). Online at: http://www.ft.dk/samling/20081/lovforslag/l181/som_vedtaget.htm

Danish Ministry of Environment and Energy, (1996). Energy 21 - the Government's energy action plan 1996 (in Danish). Online at: http://www.offshorecenter.dk/log/bibliotek/energi21.pdf

Danish Society for Nature Conservation, Danish Ornithological Society, Danish Outdoor Council, Animal Protection, Danish Anglers' Association, The Ecological Council, NOAH, (2011). Such is the country - figures about agriculture 2011 (in Danish). Online at: http://www.dn.dk/Files/Filer/Natur/ landbrug/Saadan_ligger_landet.pdf

Danish Society for Nature Conservation (2012). DN's policy on biogas - the long version (in Danish). Online at: http://www.dn.dk/Default.aspx?ID=23451\&Purge=True

Danish Society for Nature Conservation (2013). Recommendations a unique possibility for nature (Danish). Online at http://www.dn.dk/

Default.aspx?ID=31654

DONG Energy (2015). New Bio Solutions. Online at http://www.dongenergy.com/en/businessactivities/thermal-power/what-we-do/new-bio-solutions

EA Energianalyse (2009). Economic aspects of second generation bioethanol (Danish). Online at: http:// www.biobrændstof.com/media/19114/samfundsoekonomisk_analyse.pdf

Energy Supply (2011). Verdo buys Ghanesian waste wood for hundreds of millions (in Danish). Energy Supply (web based daily newsletter), 31 October 2011. Online at http://www.energy-supply.dk/ paper/view/71126/verdo_kober_ghanesisk_affaldstrae_for_trecifret_millionbelob

Erhvervsstyrelsen, (n.a.). Maabjerg Energy Concept - energy from local residual products and waste (in Danish). Online at: http://groenomstilling.erhvervsstyrelsen.dk/cases/579859

EurActiv (2012). Europe's biofuels industry slips further behind US. EurActiv, 20 November 2012. Online at: http://www.euractiv.com/climate-environment/europe-biofuels-industry-fallingnews-516130

Fargione J.E., Plevin R.J., Hill J.D. (2010). The Ecological Impact of Biofuels. The Annual Review of Ecology, Evolution, and Systematics, $\mathrm{n}^{\circ} 41$, pp. 351-377. Online at: http://www.annualreviews.org/ doi/pdf/10.1146/annurev-ecolsys-102209-144720

FERN (2011). Woody Biomass for Energy: NGO Concerns and Recommendations. Online at: http:// www.fern.org/sites/fern.org/files/NGO\%20Report\%20on\%20Biomass\%2011\%20April\%202011final.pdf)

Galik C.S., Abt R.C. (2012). The effect of assessment scale and metric selection on the greenhouse gas benefits of woody biomass. Biomass and Bioenergy, $\mathrm{n}^{\circ}$ 44, pp. 1-7.

Garud R., Karnøe P. (2010). Path dependency or path creation? Journal of Management Studies, vol ${ }^{\circ}$ $47, \mathrm{n}^{\circ} 4$, pp. $760-774$.

Geels F.W. (2004). From sectoral systems of innovation to socio-technical systems insights about dynamics and change from sociology and institutional theory. Research Policy, ${ }^{\circ}$ 33, pp. 897-920. Greenpeace (2010). In written comment to Danish Energy Projections/VE Action Plan. Online at: http://www.ens.dk/da-DK/Politik/Eus-klima-og-energipolitik/Klima-og-energipakken/ Documents/Samlede\%20h\%C3\%B8ringssvar.pdf 
Gylling M., Jørgensen U., Bentsen N.S., Kristensen I.T., Dalgaard T., Felby C., Johannsen V.K. (2012). The +10 million tons plan - potential for an increased Danish production of sustainable biomass for biorefineries (in Danish). Department of Food and Resource Economics, Faculty of Science, University of Copenhagen.

Hansen T. H. (2014). Slurry gas for the first time on the natural gas grid (in Danish). Ing.dk, 08-01-2014. Online at: http://ing.dk/artikel/gyllegas-foerste-gang-paa-naturgasnettet-165456

Henningsen J., Lidegaard M., Wenzel H. (2009). Biofuels in Danish climate policy (Danish). CONCITO.

Hermansen J.E., Knudsen M.K., Sørensen J. (2013). Soya and Palm Oil - Certification schemes for documentation of sustainability in the production. DCA rapport nr. 020, March 2013, Aarhus University. Online at: http://pure.au.dk//portal/files/68207663/

dca_rapport_Soja_og_palmeolie.pdf

Hughes T.P. (1987). Evolution of large technological systems. In W. Bijker, T.P. Hughes, T. Pinch (Eds.), The Social Construction of Technological Systems, Cambridge, MIT Press, pp. 51-82.

IDA (2009). IDA's Climate Plan 2050 (in Danish). Danish Society of Engineers. Copenhagen. Online at: http://ida.dk/publikationer/rapport/idas-klimaplan-2050

Illum K. (2014). Wood pellets versus coal - the sustainability of the green research, chronicle (in Danish). The Engineer, February 17. Online at: http://ing.dk/artikel/kronik-traepiller-versus-kulden-groenne-forsknings-baeredygtighed-166391

Jessop B. (2010). Redesigning the State, Reorienting State Power, and Rethinking the Stat. In Leicht K. T., Jenkins J. C. (eds), Handbook of Politics: State and Society in Global Perspective, Handbooks of Sociology and Social Research, Springer Science+Business Media LLC, pp. 41-61.

Jessop B. (2011). Metagovernance. In M. Bevir (ed.), The SAGE Handbook of governance, London, Sage Publications Ltd, pp. 106-123.

Jørgensen M. S., Andersen B. H. (2012). The Controversies over Bioenergy in Denmark: 'Bio' Is Not the Same as 'Sustainable'. Environmental Engineering and Management Journal, n 11, pp. 2101-2119.

Jørgensen U. (2012). Mapping and navigating transitions - The multi-level perspective compared with the arenas of development. Research Policy, $n^{\circ} 41$, pp. 996-1010.

Kuchler M., Linner B-O. (2012). Challenging the food vs. fuel dilemma: Genealogical analysis of the biofuel discourse pursued by international organizations. Food Policy, $\mathrm{n}^{\circ} 37,581 \mathrm{p}$.

Latour B. (1988). The Prince for Machines as Well as for Machinations. In Elliott B. (Ed.), Technology and Social Process, Edinburgh, Edinburgh University Press, pp. 20-43.

Latour B. (1992). Where are the Missing Masses? Sociology of a Few Mundane Artefacts. In Bijker W., Law J. (eds.), Shaping Technology, Building Society: Studies in Sociotechnical Change. Cambridge, Massachusetts, MIT Press, pp. 225-258.

Loehr-Petersen J. (2010). Earth's soil - World's gold (in Danish). In Grøn Hverdag on behalf of the Association of Biodynamic Farmers in Grøn Hverdag, 2/2010.

Lund H. (ed.) (2011). Coherent Energy and Environmental System Analysis. Final report from The CEESA project financed by The Danish Council for Strategic Research Programme Commission on Sustainable Energy and Environment. Online at http://www.ceesa.plan.aau.dk/ digitalAssets/32/32603_ceesa_final_report_samlet_02112011.pdf

Mandate for analysis of bio-energy set out in the Energy Agreement of 22 March 2012 (2012). Online at: http://www.ens.dk/sites/ens.dk/files/dokumenter/side/kommissorium.pdf

Melgaard S. (2010). NOAH's Energy Action Plan 2050. NOAH's Forlag, Denmark. 
Ministry of Climate, Housing and Energy (2014). Summary memorandum. Council meeting 13 June 2014.

Maabjerg Energy Concept (2012). About Maabjerg Energy Concept, (Danish). Online at: http:// www.maabjergenergyconcept.dk/media/1255/MEC_baggrundsnotat_presse_27april2012.pdf

Nielsen B.S., Espersen S., Nielsen J.B.H., Ravnsbæk P., Parsby M., Boldt J. (1994). Biomass for energy purposes - a strategic paper (in Danish). Reports by the Danish Board of Technology 1994/4.

NOAH (2008). NOAH's position paper on agrofuels (in Danish). Online at: http://noah.dk/wpcontent/uploads/2010/09/2008_april_NOAH_agrofuel_holdningspapir.pdf

NOAH (2010). Far too much biomass - the Climate Commission does not live up to its name (in Danish). Press release in response of the publication of the report from the Climate Commission (commissioned by the government. Online at: http://noah.dk/foedevarer/alcommissioned by the t-for-meget-biomasse-klimakommissionen-lever-ikke-op-til-sit-navn/

NOAH (2012). Our Energy but not Our Climate (in Danish) January 17, press release in response of the publication of the government's energy plan. Online at: http://noah.dk/wp-content/ uploads/2012/01/Til_Energiforhandlingerne.pdf

Nordbo J., Felby C., Grunnet K., Haaland T. (2013). Stick to knowledge - not just positions (in Danish). Newspaper comment in Politiken by WWF, University of Copenhagen, Danish Energy Association and Greenpeace.

Novozymes (2010). The Novozymes report 2010. Online at: http://report2010.novozymes.com/ service/download-report/the-novozymes-report-2010.pdf

Novozymes (2015). Laundry enzymes. Online at: http://www.novozymes.com/en/about-us/ positions-and-policies/Novozymes-positions/Pages/Detergent-enzymes.aspx

Nørgaard A.S. (2012). Development of the Danish forests as part of a sustainable Danish biomass strategy, (in Danish). BSc thesis, Department of Management Engineering, Technical University of Denmark.

Partzsch L. (2011). The legitimacy of biofuel certification. Agricultural Human Values, $\mathrm{n}^{\circ} 28$, pp. 413-425.

Pedersen T.B. (2013). Fast reforms can enable billion growth in the agriculture (in Danish). Børsen, 6 July. Online at: http://borsen.dk/nyheder/politik/artikel/1/269829/ hurtige_reformer_kan_skabe_milliardvaekst_i_landbruget.html

Politiken (2006). EU: Denmark has not focused enough on biofuels. Politiken.dk, 04-04-2006. Online at: http://politiken.dk/indland/ECE142212/eu-danmark-har-ikke-satset-nok-paa-biobraendstof/

Scarlat N., Dallemand J.-F. (2011). Recent developments of biofuels/bioenergy sustainability certification: A global overview. Energy Policy, n 39, pp. 1630-1646.

Schlegel S., Kaphengst T. (2007). European Union Policy on Bioenergy and the Role of Sustainability Criteria and Certification Systems. Journal of Agricultural \& Food Industrial Organization, vol. $5, \mathrm{n}^{\circ} 2$.

Schmidt M.(2009). Biogasreststoffe belastet den Boden. Bayrisches Landwirtschaftliches Wochenblatt, 13 November, pp. 32.

Tersbøl M. (2009). Organic biogas - why and how (Danish). Danish Agricultural Advice, The National Centre, Ecology.

The 92-group (2011). Newsletter of the 92 group (in Danish). September, $\mathrm{n}^{\circ} 75$. Online at: http:// www.92grp.dk/cms/92/nyhedsbreve/2011/Nyhedsbrev75.pdf 
The Danish Board of Technology (2009). White paper about the perspectives of biofuels in Denmark with focus on second generation bioethanol (Danish). Online at: http://www.tekno.dk/subpage.php3? article $=1602 \&$ toppic $=$ kategori7\&language $=u k$

The Danish Commission on Nature and Agriculture (2013). Nature and Agriculture - a new start. Final report (in Danish). Online at: http://www.naturoglandbrug.dk/slutrapport_2013.aspx? ID $=52071$

The Danish Ecological Council (2014). Concern over lack of transport dimensions (in Danish). Letter to Minister for Climate, Energy and Building from: 3F, Danish Agriculture \& Food Council, Federation of Danish Motorists, Danish Energy Association, DONG Energy, Novozymes, WWF, The Danish Ecological Council, Danish Oil Industry Association, BioRefining Alliance. Online at: http://www.ecocouncil.dk/udgivelser/artikler/trafik-og-luft/2302-fravaer-af-energi-ogklimamal-for-transportsektoren-i-eu

The Danish Forest Association (2011). Danish forests can double the supply of wood for energy. Presentation at audience for the Climate, Energy and Building Committee, December 15. Online at: http://www.skovforeningen.dk/media/klimaudvalget_2011-12-15.pdf

The Danish Government (2013). Growth Plan for food (in Danish). Online at: http://www.evm.dk/ / media/oem/pdf/2013/2013-publikationer/16-12-13-vaekstplan-for-foedevarer/vaekstplan-forfoedevarer.ashx

Vilby K. (2007). Biofuels, 1st and 2nd generation - risks and advantages. An analysis from the 92-group (in Danish). Online at: http://www.92grp.dk/cms/92/92grpmener/

Biodiversitet/07Biobraendstof_92_gruppen.pdf

Vinther F.P. (2011). Effects of straw mulching and straw burning on soil organic matter. Notes in Danish prepared for the Danish Ministry of Food, Agriculture and Fishery 03-11-2012, Department of Agroecology, Aarhus University. Online at: http://pure.au.dk/portal/ files/44415843/031111_AU_AGRO_Organisk_stof_ved_afbr_nding_af_stub.pdf

Wenzel H. (2013). Prioritizing biomass in the sustainable 'Smart Society'.Presentation by Henrik Wenzel, University of Southern Denmark at: 4th Generation District Heating - Second Annual Conference Aalborg University, Copenhagen, 21 August.

Wittrup S. (2014). Think tank: Biomass should be taxed as coal in power plants, article (in Danish). The Engineer (referring to the green think tank CONCITO), March 11. Online at: http:// ing.dk/artikel/taenketank-biomasse-boer-beskattes-som-kul-i-kraftvaerkerne-166955 WWF (2011). The role of Denmark in relation to the global production of palm oil (Danish). Online at: http://awsassets.wwfdk.panda.org/downloads/palmeolierapport.pdf

Zanchi G., Pena N., Bird N. (2010). Joanneum research, The upfront carbon debt of bioenergy. Joanneum Research. Online at: http://www.transportenvironment.org/sites/te/files/media/ The_upfront_carbon_debt_of_bioenergy_Joanneum_Research.pdf

\section{RÉSUMÉS}

$\mathrm{Au}$ Danemark, les controverses sur la bioénergie sont analysées sous l'angle de la transition durable et dans l'optique de soutenir l'utilisation de la bioénergie par des sociétés industrialisées dans la perspective de l'abandon d'un système reposant sur des sources fossiles. Ces analyses s'appuient sur le concept de sphère du développement (Arena of Development, AOD) pour examiner comment les acteurs interagissent et façonnent les controverses, parallèlement à 
l'exploration des processus de gouvernance en jeu. La bioénergie occupe une place centrale dans la stratégie nationale actuelle du Danemark face au changement climatique, mais certaines controverses opposent plusieurs catégories de parties prenantes à propos des sources de biomasse, des volumes et des conséquences de l'utilisation de la bioénergie pour l'environnement. Ainsi, les producteurs d'énergie convertissent les centrales au charbon à de la biomasse importée, afin que ces installations puissent continuer de jouer un rôle dans la transition vers des énergies renouvelables; de leur côté, des organisations non gouvernementales (ONG) affirment que cette pratique ne permettra pas une réduction des émissions. Les agriculteurs considèrent que le lisier permet de produire du biogaz et que cette utilisation atténue l'impact du changement climatique ainsi que les nuisances dues au lisier; à l'inverse, les ONG craignent que le biogaz issu du lisier ne serve d'argument en faveur d'une expansion de l'élevage. Une société de biotechnologie développe des enzymes pour transformer de la biomasse qui servira à produire des biocarburants de deuxième génération, et son projet a recueilli un certain soutien; en revanche, aux yeux d'autres acteurs, il ne s'agit que d'un écoblanchiment (green-washing) du système de transports actuel. Ces controverses naissent de points de vue divergents sur l'affectation durable des terres et sur les stratégies propices à l'équité dans le monde. La discussion montre comment les mécanismes de dépendance à la trajectoire et de création d'une trajectoire influencent la manière dont la bioénergie est utilisée aujourd'hui et pourrait l'être demain. Elle met au jour les processus de gouvernance liés aux controverses (décisions étatiques, création d'un marché et réseaux).

Danish bioenergy controversies are analysed from a sustainable transition perspective and as a case on industrialized societies' use of bioenergy as part of the transition away from an energy system built on fossil energy. The analyses are based on the concept of Arena of Development for analyses of how actors are interacting and shaping controversies, combined with analyses of the involved governance processes. Bioenergy has a central role in the current national Danish climate strategy, but there are controversies involving several types of stakeholders over biomass sources and amounts and the environmental impacts of using bioenergy: Energy companies convert coal fired power plants into imported biomass to sustain the role of these power plants in the transition towards renewable energy, while some NGOs claim that this practice will not reduce emissions. Farmers see slurry as a source for biogas and a measure to reduce climate impact and nuisances from slurry, while NGOs fear slurry-based biogas is used as argument for increased animal husbandry. A biotech company develops enzymes for processing of biomass for second generation biofuels, which has obtained some support, while others see this as merely "green-washing" of the present transportation system. The controversies originate in different views on sustainable land use and on strategies for global equity. The discussion identifies how mechanisms of path dependency and path creation are influencing the way bioenergy is used and proposed to be used in the future. Governance processes in relation to the controversies are identified (governmental command, market creation and networking).

\section{INDEX}

Mots-clés : sphère du développement, bioénergie, énergies renouvelables, transition, gouvernance, durabilité

Keywords : arena of development, AOD, bioenergy, transition, renewable energy, governance, sustainability

Code JEL Q48 - Government Policy, Q55 - Technological Innovation 


\section{AUTEURS}

\section{BENTE HESSELLUND ANDERSEN}

Center for Design, Innovation and Sustainable Transition, Department of Development and Planning, Université d'Aalborg, Copenhagen SV, Danemark ; arnaud.sergent@irstea.fr

\section{MICHAEL SøGAARD JøRGENSEN}

Center for Design, Innovation and Sustainable Transition, Department of Development and Planning, Université d'Aalborg, Copenhagen SV, Danemark ; msjo@plan.aau.dk 